\title{
A brief cardiac arrest due to saline irrigation during surgery for infratentorial cerebellar tumor
}

\author{
Youngsoon Kim, Chul Ho Chang, Sung Yeon Ham, and Dong Woo Han \\ Department of Anesthesiology and Pain Medicine, Gangnam Severance Hospital, Yonsei University College of Medicine, Seoul, Korea
}

The posterior fossa is surrounded by bone and tentorium, and an infratentorial brain tumor can compress normal brain structures, thus eliciting various symptoms. Surgery for an infratentorial tumor may stimulate deep brain structures, which can lead to cardiac autonomic responses. We report a brief sinus arrest immediately before dural closure following saline irrigation during removal of a cerebellar tumor.

A 38-year-old female patient with no previous medical history underwent an elective surgery for resection of an infratentorial cerebellar tumor. The patient was premedicated with glycopyrrolate $0.2 \mathrm{mg}$ and anesthesia was induced with propofol $80 \mathrm{mg}$ and rocuronium $50 \mathrm{mg}$. Anesthesia was maintained with $50 \% \mathrm{O}_{2}$ in air with $1.6-2.2 \%$ sevoflurane and infusion of remifentanil $(0.1-0.15 \mu \mathrm{g} / \mathrm{kg} / \mathrm{min})$ and rocuronium $(4.5-5.0 \mu \mathrm{g} / \mathrm{kg} /$ $\mathrm{min})$. The patient was placed in the left lateral park-bench position with the head flexed and the midline suboccipital approach was used for the surgery. The tumor was removed successfully and the dura mater was almost closed except for a $2 \mathrm{~mm}$-sized window for saline irrigation. The patient's vital signs were stable, with blood pressure 110/70 $\mathrm{mmHg}$, heart rate (HR) 68 beats per min (bpm), and peripheral oxygen saturation $\left(\mathrm{SpO}_{2}\right) 100 \%$. The end tidal carbon dioxide partial pressure $\left(\mathrm{ETCO}_{2}\right)$ was adjusted to around $32 \mathrm{mmHg}$. Normothermia was maintained at $36.0-36.2^{\circ} \mathrm{C}$ using a forced-air patient warming system (Bair Hugger ${ }^{\circledR}$ warming unit, Arizant Healthcare, MN, USA). Room temperature saline was used as an irrigation solution and $30 \mathrm{ml}$ of saline was administered in a $30 \mathrm{ml}$ syringe with a $17 \mathrm{G}$ curved needle tip through a small window of the dura mater. Instantly, the patient developed profound bradycardia and sinus arrest.
Electrocardiogram rhythm and arterial blood pressure curve showed an 8-second period of sinus arrest followed by sinus bradycardia. Immediately after being informed of the events, the surgeon stopped the irrigation. The HR returned to normal sinus rhythm (65-70 bpm) without any other treatment and the patient experienced no additional events thereafter. The patient was extubated in the intensive care unit on the following day. Her mental status recovered well and vital signs were stable.

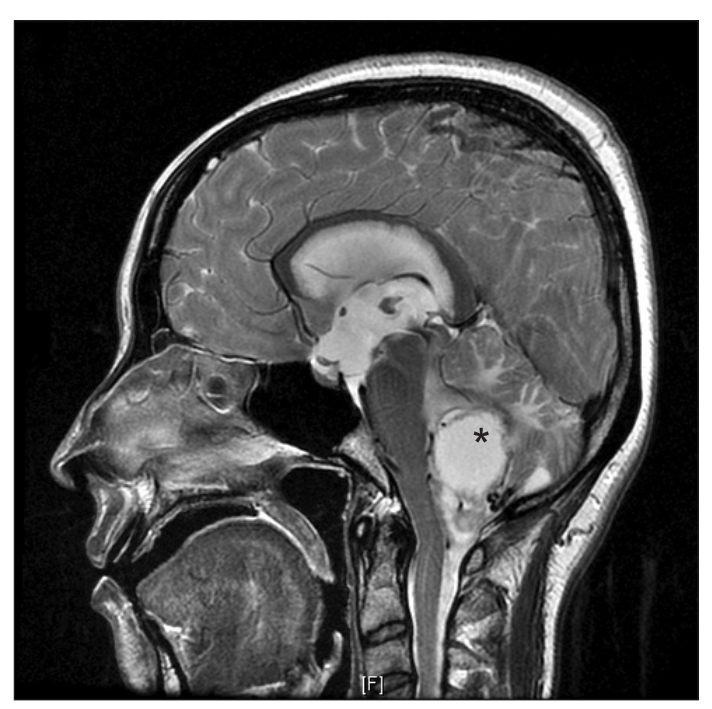

Fig. 1. Magnetic resonance image of the tumor in our case; a sagittal view. Asterisk indicates the tumor location. Multilobualr cystic lesion with the enhancing mural nodule is seen in the cerebellar paramedial portion.

Corresponding author: Dong Woo Han, M.D., Ph.D., Department of Anesthesiology and Pain Medicine, Yonsei University Gangnam Severance Hospital, 211, Eonju-ro, Gangnam-gu, Seoul 135-720, Korea. Tel: 82-2-2019-6891, Fax: 82-2-3463-0940, E-mail: hanesth@yuhs.ac

(c) This is an open-access article distributed under the terms of the Creative Commons Attribution Non-Commercial License (http:// creativecommons.org/licenses/by-nc/3.0/), which permits unrestricted non-commercial use, distribution, and reproduction in any medium, provided the original work is properly cited. 
We found one case report of three episodes of severe bradycardia and sinus arrest during anterior temporal lobectomy and amygdalo-hippocampectomy for the treatment of epilepsy [1]. The resection of the amygdala, which is the accelerator fibers controlling the HR, was the possible cause of the first episode. The remaining two episodes of sinus arrest occurred because of saline irrigation, as in our case. We suggest some possible reasons for the asystole in our case. First, elevated intracranial pressure (ICP) from excessive pressure due to irrigation resulted in bradycardia and sinus arrest. In our case, a $30 \mathrm{ml}$ syringe with a $17 \mathrm{G}$ needle tip was used and this could produce high irrigation pressure through the small sized dura opening. Increased ICP leads to pressure on and stretch of the brainstem, and it can result in hypertension, bradycardia, and apnea, which is called the Cushing reflex. Fàbregas et al. [2] suggest that excessive irrigation pressure can raise ICP during neuroendoscopy. Second, saline irrigation directly stimulated the brain stem and/or cer- ebellum. As seen in Fig. 1, the tumor was located in the cerebellar paramedial portion and the dura window for irrigation was over the lower cerebellar portion. Bradley et al. [3] described the involvement of the cerebellar uvula in cardiovascular control. Stimulation of sublobule IX-b of the posterior vermis of the cerebellum in animal models was found to produce hypotension and bradycardia by Rocha et al. [4]. Third, the local cooling effect of saline at room temperature affected the deep brain tissue. Iatrou et al. [5] suggested that cool irrigation fluid can decrease cerebral temperature and that this effect can last up to several minutes and thus can cause stimulation of the brain structures including the brain stem, thereby inducing the heart rate change.

Prompt communication with the surgeon and careful attention are necessary for early treatment of severe bradycardia, especially during saline irrigation which influences deep brain structures. Additionally, avoiding high pressure irrigation with cool saline can prevent profound bradycardia or sinus arrest.

\section{References}

1. Sinha PK, Neema PK, Manikandan S, Unnikrishnan KP, Rathod RC. Bradycardia and sinus arrest following saline irrigation of the brain during epilepsy surgery. J Neurosurg Anesthesiol 2004; 16: 160-3.

2. Fàbregas N, Valero R, Carrero E, Tercero J, Caral L, Zavala E, et al. Episodic high irrigation pressure during surgical neuroendoscopy may cause intermittent intracranial circulatory insufficiency. J Neurosurg Anesthesiol 2001; 13: 152-7.

3. Bradley DJ, Ghelarducci B, Patron JF, Spyer KM. The cardiovascular responses elicited from the posterior cerebellar cortex in the anaesthetized and decerebrate rabbit. J Physiol 1987; 383: 537-50.

4. Rocha I, Goncalves V, Bettencourt MJ, Silva-Carvalho L. Effect of stimulation of sublobule IX-b of the cerebellar vermis on cardiac function. Physiol Res 2008; 57: 701-7.

5. Iatrou CC, Domaingue CM, Thomas RD, Nye DH. The effect of selective brain cooling on intracerebral temperature during craniotomy. Anaesth Intensive Care 2002; 30: 167-70. 